\title{
Assessment of fatigue damage in low-carbon steel using Lock-In-Thermography
}

\author{
by J. Medgenberg*, T. Ummenhofer*
}

*Institut fuer Bauwerkserhaltung und Tragwerk, Technische Universitaet CaroloWilhelmina, Braunschweig, Pockelsstrasse 3, 38106 Braunschweig/Germany

\begin{abstract}
This paper presents experimental results about the thermographic detection of localized cyclic-plasticity in early stages of fatigue damage accumulation of lowcarbon steel. Thermoelastic and thermoplastic temperature variations are separated by a special data processing technique. For fatigue loaded specimens the extension and the magnitude of cyclic plasticity within the hot spots shows a strong connection to local fatigue life and crack initiation. The thermographic results are compared with microscopic pictures taken from the specimen surface which confirm plastic activities. The results show sensitivity towards the individual microstructural circumstances of the used specimens. A major advantage is the capability to assess local fatigue damage from a relatively macroscopic point of view.
\end{abstract}

\section{Introduction}

Most testing methods concentrate on the detection of fatigue cracks and fail to analyse and quantify early stages of fatigue damage accumulation. Depending on the material and the loading conditions a long time span may exist, for which the accumulated fatigue damage can therefore not be assessed experimentally. Since fatigue damage accumulation, plasticity induced damping of mechanical energy and intrinsic heat generation are strongly interdependent, infrared imaging seems to be a very promising tool for the assessment of early fatigue damage in steel components.

High cycle fatigue occurs if structures or structural details are exposed to cyclic loading at relatively low load amplitudes. For the low-carbon steel treated within this paper stress levels typically stay well beyond the static yield limit and hundreds of thousands up to millions of load cycles can be applied until a macroscopic crack can be detected. Even though the structure macroscopically still behaves completely elastic, the cyclic yield limit may be exceeded in the critical hot spots of the structure as e.g. welding seams and geometric notches. Within these spots the repeated load cycles lead to inhomogeneous plastic cyclic deformations at a microscopic scale. It first manifests in several unfavourable orientated grains within the metal matrix in form of complex dislocation generation and interaction processes. As a result of these processes, the development of persistent slip bands can be observed at the surface. In course of the further fatigue process these slip bands deepen and might become the initiation points of microcracks. Eventually a macroscopic crack evolves from the weakest point of the fatigued metal matrix and grows until the structure fails completely.

Cyclic plasticity is often either investigated from a completely macroscopic point of view or research concentrates on the microscopic characterization of the underlying processes. The aim of this paper is to demonstrate that thermography offers high potential as a testing method for the fatigue damage assessment in the mesoscopic range, i.e. for the characterization of the processes within the hot spots of structural details. 


\section{Thermoelastic and thermoplastic temperature variations}

\subsection{Background}

The thermoelastic effect relates the first invariant of the elastic strain tensor, i.e. the elastic volume change, of a solid body under mechanical loading to the generation of heat within the body. Reversely, the local temperature change of the structure under mechanical loading is connected to its internal strain and stress state. This allows for an experimental strain analysis by measuring the small temperature changes during mechanical loading. The theoretical base for this method was laid in the 19th century. Along with the practical implementation for experimental strain analysis in the 20th century the method has been termed thermoelastic strain (stress) analysis (TSA).

TSA focuses on the quantitative measurement of temperature changes of periodically loaded structures under the assumption of fully reversible, respective linear-elastic material behaviour. In this case the net heat generation over a complete load cycle equals zero and the mean temperature of the loaded body remains unchanged.

As outlined before, the fatigue damage accumulation of metals is tightly linked to inelastic material behaviour, primarily due to the onset of plasticity [1]. This is accompanied by strong damping of induced mechanical energy due to plasticity. From experimental results it has been concluded that most of the dissipated energy during plastic deformation is released as heat and only a fraction of $5 \%$ to $10 \%$ is converted into irreversible structural changes within the material. Further thermomechanical considerations indicate that the ratio between the stored energy and the energy converted to heat is not a constant, but depends on the loading level and the loading history [11]. In contrast to the thermoelastic effect, damping due to plasticity leads to a net heat generation. This causes a continuous heating-up of a periodically loaded body until it reaches a dynamic thermal equilibrium with its surrounding. However, since for high cycle fatigue cyclic plasticity is limited to local stress concentrations the global mean temperature may not be a sufficiently good damage indicator for the lifetime assessment of complex structures. Consequently our approach focuses on the thermographic detection of characteristic changes in the local thermoelastic signal which are less dependent on the individual constraints.

The 3-dimensional heat conduction equation including thermoelastic coupling (Eq.1) can be derived from the first and second law of thermodynamics. For the derivation (see e.g. [10]) reversibility, linear elastic material behaviour and isotropic and constant material coefficients are assumed. Neglecting any higher order coupling terms, the effects of plasticity are included phenomenologically by taking the plastic work rate $\dot{W}_{P}$ as an additive source term into the thermoelastic equation

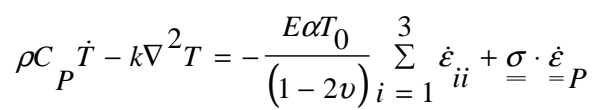

In Eq.1 $\rho$ denotes the density, $\mathrm{C}_{\mathrm{P}}$ is the heat capacity at constant pressure, $\mathrm{T}$ the temperature, $\mathrm{k}$ the thermal conductivity, $\mathrm{E}$ the Young's modulus, $\alpha$ the coefficient of thermal expansion, $T_{0}$ the reference temperature under the assumption of small temperature changes, $v$ the Poisson's ratio and $\varepsilon_{\mathrm{ii}}$ the elastic strain tensor. The second term on the right hand side is the plastic work rate where $\underline{\underline{\sigma}}$ is the stress tensor and $\underline{\underline{\varepsilon}} P$ is the plastic strain tensor. The dot denotes the time derivative. 


\subsection{Numerical and experimental studies}

Figure 1 shows the hourglass-shaped specimen-type used throughout the studies for this paper. The centre part of the specimen contains an additional mild notch with a geometric shape factor of 1.36. The notch acts as a local stress riser to ensure localized fatigue damage accumulation. The region just below and above the notch has been flattened to prevent loss of focus during observation with the infrared camera.
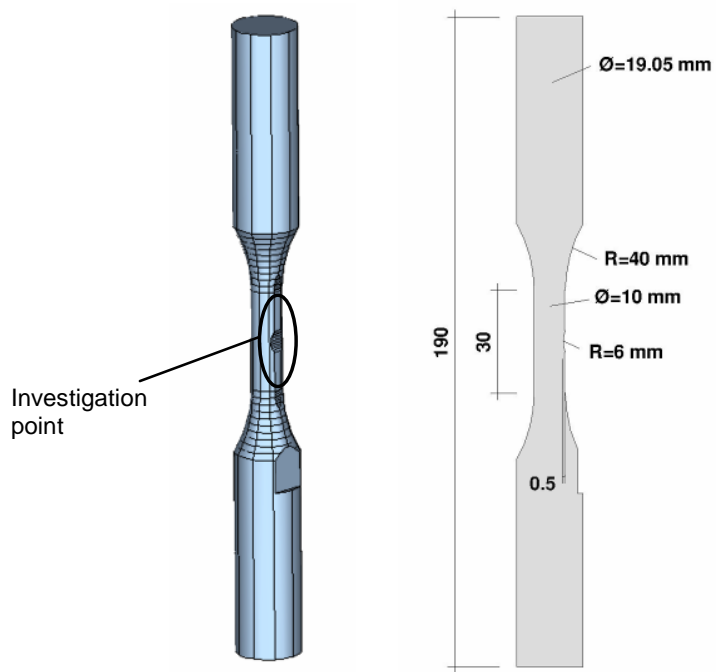

Fig 1. Specimen type used for numerical and experimental studies

Assuming a plasticity model and solving Eq. 1 numerically, the thermoelastic and thermoplastic temperature variations of the specimen under mechanical loading can be calculated. In order to study the influence of plasticity on the local thermoelastic temperature evolution we performed a series of numerical simulations with different loading conditions. The procedure and some results are reported in [12]. Figure 2, left shows the calculated temperature evolution at the mid point of the notch under sinusoidal loading for purely linear elastic and elastic-plastic material behaviour. The overall shape of the thermoelastic temperature variation follows the sinusoidal mechanical loading. The temperature variation due to thermoelasticity is negative for tension and positive for compression half-cycles. Figure 2, right shows the difference in temperature between the linear-elastic model and the elastic-plastic model. The temperature difference between both models is always positive, due to the additive plastic heat generation rate in Eq. 1. The linear trend in the temperature originates from the global warming-up of the numerical model since a steady-state solution could not be reached for a reasonable number of calculations. The temperature difference between the two models becomes higher for tension than for compression half cycles. This can be explained by the decomposition of the strain tensor in Eq. 1 in elastic and plastic strains. If the yield criterion is exceeded within the numerical model the onset of plasticity causes a decrease of the elastic strain rate which leads to a reduced thermoelastic temperature variation. The summation with the thermoplastic temperature changes gives the shown characteristics. 

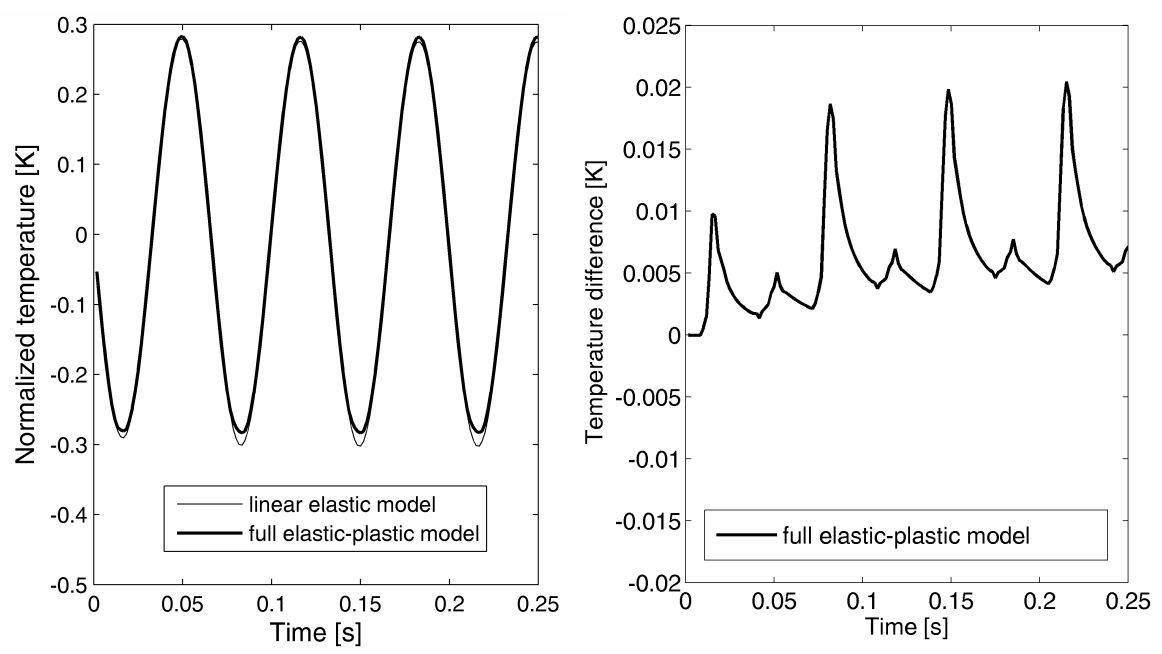

Fig 2. Calculated temperature variation at the central point of the notch for a linearelastic and an elastic-plastic numerical model and temperature difference.
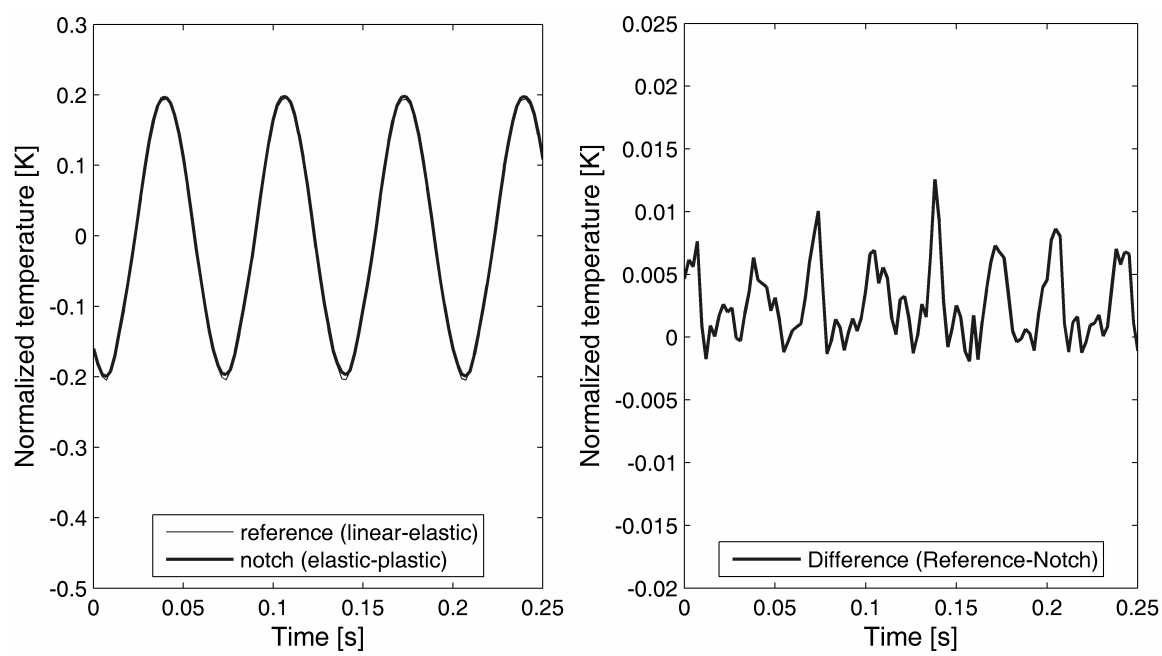

Fig 3. Measured temperature variations and difference signal

Figure 3, left shows similar experimental results obtained from a fatigue loaded specimen for which the cyclic yield limit is locally exceeded within the notch. The thermoelastic temperature variations are obtained from a point near the notch where purely elastic behaviour can be assumed. The results have been obtained after numerous load cycles i.e. for a steady state of the specimen. Figure 3, right gives the difference between the thermoelastic temperature signal and the temperature signal within the notch. Note that the experimentally obtained difference signal qualitatively resembles strongly the numerically calculated thermoplastic temperature evolution in figure 2 right. 
The results demonstrate that cyclic plasticity leads to characteristic changes of the thermoelastic temperature evolution which are non-uniformly distributed both in time and space. More work is needed to improve the quantitative consistency of numerical and experimental results.

\section{Experimental detection of cyclic plasticity during high cycle fatigue}

\subsection{Setup and testing conditions}

In order to characterize the evolution of localized cyclic plasticity during high cycle fatigue a series of load-controlled fatigue tests were performed on the specimen type shown in figure 1. For this purpose a special setup (figure 2) has been installed in front of a servohydraulic testing machine (MTS 200). The equipment comprises a 3-axial positioning system which carries a video-light-microscope and an infrared camera. The positioning system and both cameras can be triggered via the testing machine so that fully automated scan procedures can be accomplished.

The system allows for in situ light-microscopic and thermographic investigations of a specimen surface during fatigue tests which gives the opportunity to compare thermographic results with microscopically detectable damage processes.
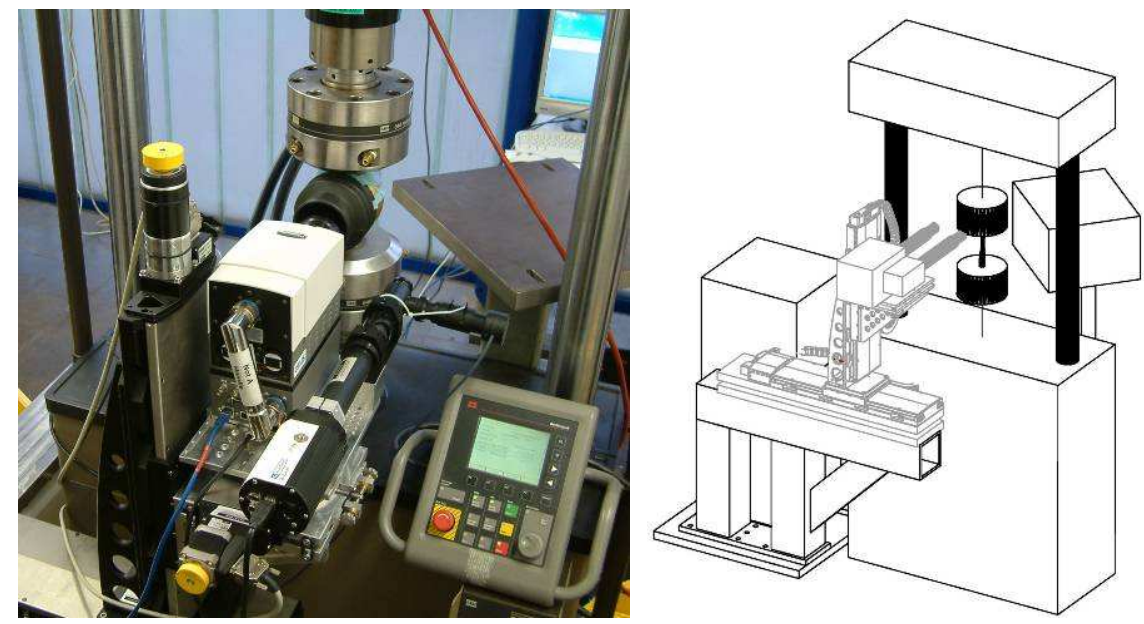

Fig 4. Test setup for in-situ thermograpic and light-microscopic investigations

All specimens have been mechanically worked out of a plane steel plate with a thickness of $20 \mathrm{~mm}$. The direction of rolling of the steel-plate coincides with the longitudinal axis of the specimen. The material is a low carbon ferritic-pearlitic steel S355J2G3 which is commonly used as general-purpose construction steel in civil and mechanical engineering. The upper and lower yield limit have been determined as mean from five tension tests and are $461 \mathrm{MPa}$ and $362 \mathrm{MPa}$, respectively. The mean value of the ultimate strength is $568 \mathrm{MPa}$ and the mean Youngs-Modulus is 216,000 $\mathrm{MPa}$. After manufacturing the specimens were annealed under an argon atmosphere at $600^{\circ} \mathrm{C}$ for one hour in order to relieve any resid ual stresses. Then the specimens were polished mechanically with diamond polish paste of decreasing coarseness down to $1 \mu \mathrm{m}$. Possible residual stresses from the mechanical polishing procedure 
are expected to be negligible for our purposes. In order to allow for an easier interpretation of the microscopic pictures, the steel surface around the notch has been slightly etched for a couple of seconds with a 3\% Nital solution. Afterwards the specimens were coated with a high emissivity coating in order to ensure a high and homogenous emissivity of the polished steel surface for the thermographic measurements. A small part of the notch region has been left uncoated for additional microscopic investigation.

The fatigue tests were performed with a loading frequency $f_{L}=15 \mathrm{~Hz}$ and a load ratio $R=-1$, i.e. fully reversed high cycle fatigue tests. For all load amplitudes the maximum principal stress in the notch region obtained from finite elements calculations did not exceed $85 \%$ of the static yield limit $\mathrm{f}_{\mathrm{y}}$. Depending on the loading conditions the fatigue loading was automatically interrupted every $15,000,20,000$ or 30,000 load steps and a microscopic scan of the uncoated specimen surface was triggered by the testing machine. Just before every interruption a sequence of 2,000 infrared pictures under fatigue loading were taken from the notch region.

The fatigue tests were finished after $2.5^{*} 10^{6}\left(1.3^{*} 10^{6}\right.$ for specimen RK0621) load cycles or if the preset maximum deformation limit of the testing machine was exceeded due to the rupture of the specimen. Table 1 summarizes the testing conditions for the individual specimens. The arrow denotes tests which have been finished before the rupture of the specimen.

Table 1. Summary of testing conditions for the individual specimens

\begin{tabular}{|c|c|c|c|c|}
\hline $\begin{array}{c}\text { Specimen } \\
\text { number }\end{array}$ & $\begin{array}{c}\text { Amplitude of } \\
\text { loading [kN] }\end{array}$ & $\begin{array}{c}\text { Amplitude of max. } \\
\text { principal stress [MPa] }\end{array}$ & $\begin{array}{c}\text { Investigation } \\
\text { interval [LC] }\end{array}$ & $\begin{array}{c}\text { Totally applied } \\
\text { load cycles } \mathbf{~}_{\mathbf{f}} \text { [LC] }\end{array}$ \\
\hline RK0614 & 16.50 & 288.9 & 30,000 & $2,500,000 \rightarrow$ \\
\hline RK0621 & 16.50 & 292.5 & 20,000 & $1,300,000 \rightarrow$ \\
\hline RK0617 & 17.00 & 295.6 & 15,000 & 783,384 \\
\hline RK0608 & 17.25 & 299.8 & 15,000 & 711,541 \\
\hline RK0625 & 17.50 & 306.8 & 15,000 & 486,932 \\
\hline RK0626 & 17.75 & 306.6 & 15,000 & 388,691 \\
\hline RK0618 & 18.00 & 310.5 & 15,000 & 477,135 \\
\hline RK0631 & 18.25 & 311.0 & 15,000 & 256,054 \\
\hline RK0624 & 18.50 & 324.1 & 15,000 & 218,180 \\
\hline
\end{tabular}

\subsection{Data processing}

The camera type used for the thermographic investigation is a FLIR Phoenix with a $640 \times 512$ InSb detector. The camera has been used in a subframe-mode with an integration time of $2 \mathrm{~ms}$, resulting in an effective framerate of $f_{I R}=412.5 \mathrm{~Hz}$. As seen before the plasticity-induced variations of the thermoelastic temperature signal are in the range of a few $\mathrm{mK}$, i.e. within the noise range of typical IR-detectors. Advanced data processing is therefore crucial for the detection of the small thermoplastic temperature signals.

First the detector non-uniformity is corrected using a two point gain and offset approach. The offset values are automatically updated for each measurement using a black alumina plate at room temperature. The corrected gray-values are then converted into temperature values using a calibration curve obtained from a black body.

Due to the mechanical loading within the testing machine, the investigated specimen surface moves relative - prevailing parallel - to the position of the infrared camera. Therefore successive pixels of the recorded picture stream do not belong to the same surface position of the specimen. In order to reduce the motion blur a 
compensation scheme has been used before further data processing. We assumed a purely rigid body motion between the individual pictures of the stream and aligned each picture to match a reference template taken from the unloaded specimen. The correction scheme is based on normalized cross-correlation and uses an interpolation-scheme which allows for sub-pixel accuracy.

As shown in chapter 2 the temperature variations due to plasticity lead to localized nonlinearities within the thermoelastic temperature evolution. Due to the periodicity of the signal a temporal discrete Fourier-Transform (DFT) is a suitable tool for the separation of linear thermoelastic and nonlinear thermoplastic effects. The thermoelastic temperature variation is concentrated at the loading frequency $f_{L}$ whereas plasticity gives signal contributions at $2 \mathrm{f}_{\mathrm{L}}$ and further harmonics. Let $T(t)_{x, y}$ be an infrared picture stream of $N$ individual pictures of size $x, y$ recorded with a framerate of $f_{I R}$. Then the pixelwise DFT is defined as:

$$
\mathrm{T}\left(\frac{n f_{I R}}{N}\right)_{x, y}=\sum_{k=0}^{N-1} T(k)_{x, y} e^{-j 2 \pi k n / N}
$$

The amplitude $A_{x, y}$ and phase $P_{x, y}$ can be calculated from:

$$
\begin{aligned}
& A\left(\frac{n f_{I R}}{N}\right)_{x, y}=\frac{2}{N} \sqrt{\operatorname{real}\left(\mathrm{T}\left(\frac{n f_{I R}}{N}\right)_{x, y}\right)^{2}+\operatorname{imag}\left(\mathrm{T}\left(\frac{n f_{I R}}{N}\right)_{x, y}\right)^{2}} \\
& P\left(\frac{n f_{I R}}{N}\right)_{x, y}=\tan ^{-1}\left(\frac{\operatorname{imag}\left(\mathrm{T}\left(\frac{n f_{I R}}{N}\right)_{x, y}\right)}{\operatorname{real}\left(\mathrm{T}\left(\frac{n f_{I R}}{N}\right)_{x, y}\right)}\right)
\end{aligned}
$$

Applying Eq. 2 to the measured thermoelastic data we found significant nonlinearities in the temperature evolution even in the absence of cyclic plasticity. Even though the effect is negligible for the determination of the thermoelastic temperature amplitude $\mathrm{T}_{\text {Elast }}$ by Eq. 3 , the second harmonic is strongly disturbed by this effect. Further work is in progress in order to quantify and explain the observed inconsistencies. In order to avoid the problems a new data processing method in the time domain has been developed for the separation of thermoelastic and thermoplastic temperature variations. The procedure filters out global nonlinearities within the thermoelastic temperature signal under the assumption of a purely linear behaviour between adjacent points. First a reference signal needs to be defined within the recorded pictures from a region of the specimen with supposed purely thermoelastic behaviour. This reference signal is fitted both in phase and amplitude to the temperature evolution of each pixel $x, y$ using a linear least-square approach. Then the pixelwise difference signal $\Delta T(t)_{x, y}$ between the temperature signal $T(t)_{x, y}$ and the fitted elastic reference signal $\mathrm{T}(t)_{\text {ref }}$ is calculated.

$$
\Delta \mathrm{T}(t)_{x, y}=\mathrm{T}(t)_{x, y}-T(t)_{r e f}
$$


The remaining temperature difference contains the thermoplastic signal contributions (compare to figures 2 and 3 ) which can be further separated from the detector noise by applying the DFT (Eq.2) on the remaining difference signal $\Delta \mathrm{T}(t)_{x, y}$. This value is referred throughout this paper as plastic temperature amplitude $T_{\text {Plast. }}$

\subsection{Results}

For three different specimens figure 5 shows microscopic pictures of the notch region and the dimensionless ratio $T_{\text {Plast }} / T_{\text {Elast }}$ at different relative lifetimes $N / N_{f} . N_{f}$ denotes the overall applied load cycles according to table 1. The white ellipse within the thermographic pictures covers the blank used for microscopic investigation, where the thermographic measurements are highly disturbed due too poor emissivity. Note that the scale of the microscopic pictures and the thermografic results is different.

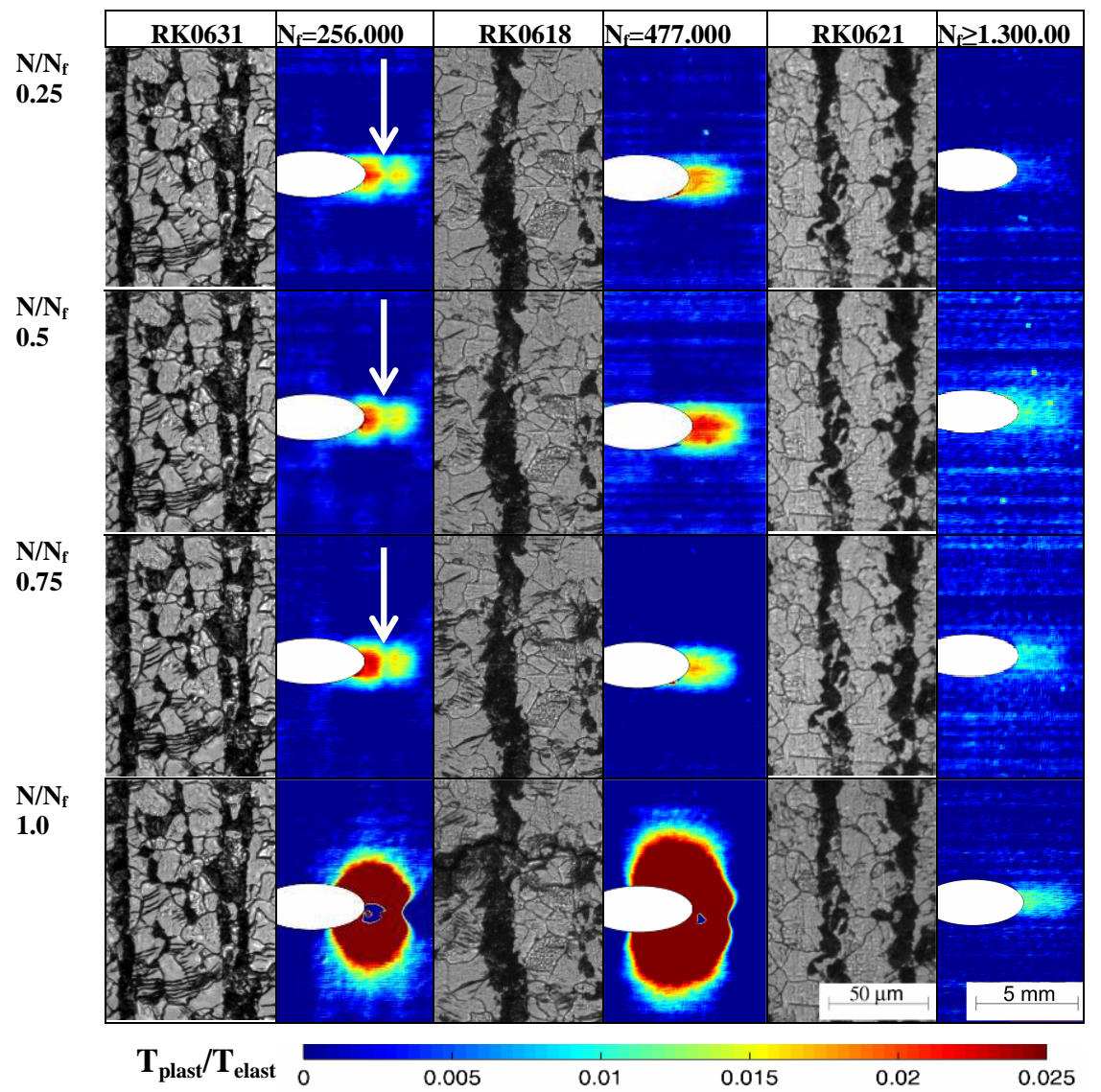

Fig 5. Microscopic surface pictures of the notch surface temperature ratio $T_{\text {plast }} / T_{\text {elast }}$ for three different specimens at different relative lifetime $N / N_{. f}$ 
From the microscopic pictures it becomes obvious that for all load levels slip bands can be found in individual grains which indicate significant cyclic plasticity. Due to the poor contrast of the pictures for the darkened pearlitic grains we could not conclude whether slip bands are exclusively confined to ferritic grains. The number of grains containing slip bands increases with increasing load amplitude and increasing load cycles. Notice that slip bands can also be found for specimens which did not fail during the fatigue tests. Alike the microscopic observations the thermographic results show a significantly higher $T_{\text {Plast }} / T_{\text {Elast }}$ ratio within the notch region. For the rest of the specimen the thermograpic result indicate a purely thermoelastic behaviour. Crack initiation leads to a strong increase of the thermoplastic temperature variations owing to the concentration of plasticity around the crack tip. Due to the small extension of the specimens and the load-control the period of crack propagation is short compared to the time span needed for crack initiation.

From the thermographic results of the specimen RK0631 it becomes obvious that the observed plastic deformations within the notch are not necessarily evenly distributed. The results of this specimen indicate a zone of decreased plastic activity. After removing the high-emissivity coating upon completion of the fatigue tests, we found a concentration of perlitic grains in this region (figure 6). We therefore conclude that the thermographic results capture the decreased cyclic plastic activities in this part of the specimen which demonstrates the high overall spatial resolution of the approach and its sensitivity towards individual microstructural circumstances.

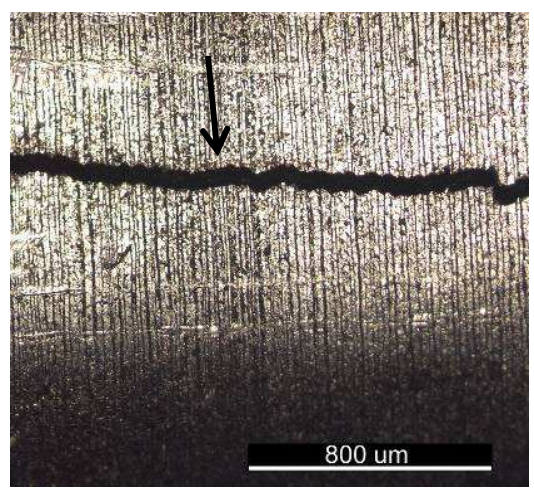

Fig 6. Concentration of pearlitic grains in specimen RK0631
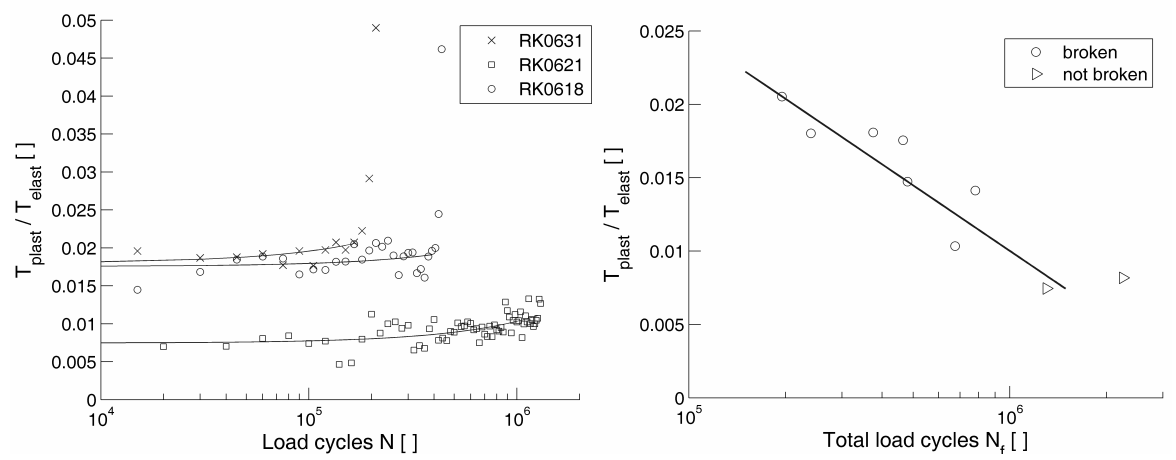

Fig 7. Evolution of the $T_{\text {Plast }} / T_{\text {Elast }}$ ratio for three different specimens over the number of load cycles and plateau value of $T_{\text {Plast }} / T_{\text {Elast }}$ as function of total lifetime. 
Figure 7 , left shows the temporal evolution of the temperature ratio $T_{\text {Plast }} / T_{\text {Elast }}$ over the course of the fatigue tests. Each point represents an individual measurement and has been obtained as a spatial mean-value of $10 \times 20$ pixels, (approximately $0.5 \times 1.0 \mathrm{~mm}$ ), within the notch. The lines give a least square-fit to the data assuming a linear variation until crack initiation. For most specimens a characteristic plateau value is reached after less than $10 \%$ of the total lifetime $\mathrm{N}_{\mathrm{f}}$. Upon crack initiation the ratio increases strongly. The plateau value remains quite constant for most of the fatigue life and increases with increasing load level, respectively decreasing total lifetime.

In Figure 7 , right the plateau value of $\mathrm{T}_{\text {Plast }} / T_{\text {Elast }}$ for all tested specimens has been plotted against their total lifetime $\mathrm{N}_{\mathrm{f}}$. It can be seen that there is a tide relation between the ratio $T_{\text {Plast }} / T_{\text {Elast }}$ and the lifetime of the specimens.

\section{Conclusions}

The preceding article presents experimental results of the thermographic detection of early fatigue damage during high cycle fatigue in low-carbon steels. From numerical studies of thermoelastic and thermoplastic behaviour, microscopic and thermographic results it has been concluded that cyclic plasticity as an early stage of fatigue damage accumulation can be localized and - to some extent quantified by thermography. In contrast to most reported results local plasticity has been detected by separating the local plasticity-induced temperature nonlinearities from the thermoelastic temperature evolution during a steady state of the loaded specimen. The method shows high resolution of spatial details and is able to resolve strongly localized phenomena within the material. Thermographic assessment of local plasticity might provide a valuable tool for the prediction of local damage evolution.

From the results a dependence of the magnitude of the detected thermoplastic temperature variations to the total lifetime has been found. The results indicate that there is a plateau in the local ratio of thermoplastic to thermoelastic temperature contributions which arises early during the fatigue process. This agrees with reported findings of the macroscopic cyclic behaviour for low-carbon steels. The magnitude of the plateau increases with increasing load amplitude and decreasing fatigue life, respectively. Further analysis of the relationship of thermoplasticity and fatigue damage accumulation can eventually allow to predict the local damage evolution for complex structures.

\section{ACKNOWLEDGEMENTS}

The authors would like to thank the German Research Foundation DFG for the funding of these studies within the framework of the collaborative research centre SFB 477 at the Technical University Carolo-Wilhelmina Braunschweig/ Germany. 


\section{REFERENCES}

[1] Bily M., Cyclic Deformation and Fatigue of Metals, Engineering Materials Science Monographs 78, Amsterdam, Elsevier Science Publishers, 1993.

[2] Boulanger T., Chrysochoos A., Mabru C., Galtier A., "Calorimetric analysis of dissipative and thermoelastic effects associated with the fatigue behavior of steels", International Journal of Fatigue, vol. 26, 2004, pp. 221-229.

[3] Charkaluk E., Constantinescu A., "Dissipation and fatigue damage", MP Materialpruefung, vol. 46, no. 10, 2004, pp. 524-530.

[4] Chrysochoos A., Muracciole J.M., Wattrisse B., "Experimental analysis of strain and damage localization", Continuous Damage and Fracture, Cachan, October 23-27, 2000, Elsevier, pp. 41-51.

[5] Harwood N. Cummings W.M., Thermoelastic Stress Analysis, IOP Publishing Ltd, Adam Hilger imprint, Bristol, 1991.

[6] Krapez J., Pacou D., "Thermography detection of early thermal effects during fatigue tests of steel and aluminium samples" AIP Conference Proceedings, Brunswick, Maine, 29 July-3 August 2001, AIP Conference Proceedings, Volume 615, pp. 1545-1552

[7] La Rosa G., Risitano, A., "Thermographic methodology for rapid determination of the fatigue limit of materials and mechanical components", International Journal of Fatigue, vol. 22, 2000, pp. 65-73.

[8] Liaw P.K., Wang H., Jiang L., Yang B., Huang J.Y., Kuo R.C., Huang J.G., "Thermographic detection of fatigue damage of pressure vessel steels at 1000 $\mathrm{Hz}$ and $20 \mathrm{~Hz}$ ", Scripta Materialia, vol. 42, 2000, pp. 389-395.

[9] Luong M.P., "Fatigue limit evaluation of metals using an infrared thermographic technique", Mechanics of Materials, vol. 28, 1998, pp. 155-163.

[10] Nowinski J.L., Theory of thermoelasticty with applications, Sijthoff \& Noordhoff Internation Puplishers B.V., Alphen aan den Rijn, 1978.

[11] Rosakis P., Rosakis A.J., Ravichandran G., Hodowany J., "A thermodynamic internal variable model for the partition of plastic work into heat and stored energy in metals", Journal of Mechanics and Physics of Solids, vol. 48, 2000, pp. 581-607.

[12] Ummenhofer, T., Medgenberg, J., 2006. "Numerical modelling of thermoelasticity and plasticity in fatigue-loaded low carbon steels", submitted for Publication in Qirt Journal, Vol.2/2006. 